\title{
On Design Analysis of Linear Active Disturbance Rejection Control for Uncertain System
}

\author{
Shangyao Shi, Jun Li and Shiping Zhao \\ School of Manufacturing Science and Engineering, Sichuan University, Chengdu \\ 610041, P. R. China \\ peter.shi@126.com,lijunj08@163.com,spzhao@scu.edu.cn
}

\begin{abstract}
It has been shown that the active disturbance rejection control (ADRC), first proposed by Han Jingqing, is very an effective approach to control the uncertain system, whether the plant is linear or nonlinear, time-varying or time-invariant. This paper has quantitatively presented the relation between the observer bandwidth of LESO and the controller's sampling-period in theory. Moreover, it is proven that the shorter the sampling-period, the higher the control degree of accuracy is for the same plant, and the smaller the plant's "time-scaling", the larger the closed-loop control error is for the same sampling-period of controller by simulation. In general, this paper will contribute to improving specific ADRC based controller design and tuning for researchers and practice engineers.
\end{abstract}

Keywords: ADRC, LADRC, LESO, sampling-period

\section{Introduction}

The active disturbance rejection control (ADRC) [1] was first proposed by Prof. Han in 1998, and after the extended state observer (ESO) [2] was presented to address estimating the uncertain disturbance [2]. Soon after, many scholars have been devoting themselves to the study of the ADRC, because it is very an effective approach to control the uncertain system, whether the plant is linear or nonlinear, time-varying or time-invariant [3] with high robustness [4-5]. Several years later, linear active disturbance rejection control (LADRC) [4], using linear extended state observer (LESO) [6] with a linear feedback, was proposed by Prof. Gao in 2003. Since then, it is applied in many industrial fields, and shows promising control performance in practice.

Although the technology of ADRC was detailed formulated in [3], its theoretical proofs were not strictly proved and seemed untenable a few years ago. Recently, some breakthroughs of theoretical researches on ADRC were obtained, such as in [5], a rigorous mathematical proof under some additional conditions has been given for the convergence of nonlinear tracking differentiator, and the weak convergence of a finite-time stable based system nonlinear high-gain tracking differentiator is presented in [7] under some loose conditions instead of the strict conditions required by the Lyapunov function. Specially, the extended state observer, which is the key link of ADRC can real-time estimate the total disturbance effecting on the system, and then adopt dynamic compensation to make the system become the canonical integral series. This paper [8] has given the rigorous proof of asymptotical convergence of ESO with total disturbance error eliminated by the high-gain. And it was proven in [9] that the convergence of non-linear extended state observer (ESO) for a class of multi-input multi-output non-linear systems with uncertainty can be feasible if the 
non-linear function for observer is properly constructed. The theoretical principle of ADRC was almost completely proven until the full-global and semi-global convergence of the nonlinear ADRC for a class of MIMO nonlinear systems with large uncertainty are proved in [10]. The stabilization of the closed-loop system were studied and compared in [11-12] by adopting the sliding mode control and active disturbance rejection control, respectively. The research in [13] showed that estimation and tracking errors is bounded, with their bounds monotonously decreasing with their respective bandwidths for large dynamic uncertainties. And the frequency-domain analysis of such a class control system is performed in [14-15] to shows that the ADRC based closed-loop system possesses a high level of robustness, whose bandwidth, stability margins and crossover frequency are nearly unchanged when the plant Parameters and input disturbance vary.

All above papers just theoretically and qualitatively analysis ADRC or its ingredient, so this paper will make an offer to quantitatively analyses the latent relationship between parameters of LESO of LADRC based controller and the sample-period of the specific controller based on LADRC under some constraint conditions. It is proven that the observer bandwidth is in proportion to the sampling-period of the controller if the sampling-period is used as the integral-time step length. Furthermore, this paper also analyses the control precision which is proven to be limited by the sampling-time and the "time-scaling" of the plant in simulations. Following the principia described in this paper, the researchers and engineers will design some outstanding and promising controller based on LADRC.

\section{The Relation between the Sampling-period and the Bandwidth of LESO}

In general case, considering a nth order system, which may be nonlinear and time-varying, with a uncertain disturbance as follow

$$
\left\{\begin{array}{l}
\dot{x}_{1}=x_{2} \\
\vdots \\
\dot{x}_{n}=f(X, w, t)+b u \\
y=x_{1}
\end{array}\right.
$$

Where $X$ is the system state variable vector $\left[x_{1} x_{2} \cdots x_{n}\right]^{T}, u$ is the control output of the controller, and $\mathrm{y}$ is the measure output. $f(\cdot)$ is a uncertain generalized disturbance including the internal disturbance and the external disturbance.

As we know that the corresponding linear extended state observer (LESO) of above system (1) with differential form, is constructed as follow

$$
\left\{\begin{array}{l}
\dot{z}_{1}=z_{2}+\beta_{1} e \\
\vdots \\
\dot{z}_{n}=z_{n+1}+\beta_{n} e+b u \\
\dot{z}_{n+1}=\beta_{n+1} e \\
y=x_{1}
\end{array}\right.
$$

Where $e=y-z_{1}, z_{1}, z_{2}, \ldots$, and $z_{n}$ are the estimates of state variables $x_{1}, x_{2}, \ldots$ and $x_{n}$, respectively, while the variable $z_{n+1}$, namely known as an augmented state variable, is the estimate of $f(\cdot)$, also named the total disturbance. 
Assumption 1: The sampling-period of controller is used as the integral-time step length, and $f(X, w, t)$ in (1) is bounded, continuous and differentiable.

Assumption 2: The LESO's parameters, denoted as $\beta_{1}, \beta_{2}, \cdots, \beta_{n+1}$, are properly selected for the LADRC based controller with the sampling-period $\tau_{1}$.

Theorem 2.1: Satisfying Assumption 1 and Assumption2, then the parameters, i.e. $\beta_{1}^{\prime}, \beta_{2}^{\prime}, \cdots, \beta_{n+1}^{\prime}$ of the LESO (2) for other controller whose sampling-period is $\tau_{2}=m \tau_{1}$, can be selected by following expression as

$$
\beta_{i}^{\prime}=\frac{\beta_{i}}{m^{i}} \quad(i=1,2, \cdots, n+1)
$$

Proof: Corresponding to the LESO (2), the discrete-time linear extended state observer (DLESO) of the system (1) can be reconstructed as

$$
\left\{\begin{array}{l}
z_{1}(k+1)=z_{1}(k)+z_{2}(k) \tau+\beta_{1} e \tau \\
\vdots \\
z_{n}(k+1)=z_{n}(k)+z_{n+1}(k) \tau+\beta_{n} e \tau+b u \tau \\
z_{n+1}(k+1)=z_{n+1}(k)+\beta_{n+1} e \tau \\
y=x_{1}(k+1)
\end{array}\right.
$$

Where $\tau$ is the integral-time step length, $z_{i}(k)$ means the value of $z_{i}(t)$ at $t=k \tau$ (where $i$ is $1,2, \ldots, \mathrm{n})$. Here, supposing the sampling-period $\tau_{1}$ is used as the integral-time step length of DLESO above, thus the specific DLESO of the controller obtained from (4) is

$$
\left\{\begin{array}{l}
z_{1}(k+1)=z_{1}(k)+z_{2}(k) \tau_{1}+\beta_{1} e \tau_{1} \\
\vdots \\
z_{n}(k+1)=z_{n}(k)+z_{n+1}(k) \tau_{1}+\beta_{n} e \tau_{1}+b u \tau_{1} \\
z_{n+1}(k+1)=a_{n+1}(k)+\beta_{n+1} e \tau_{1}
\end{array}\right.
$$

Similarly, the sampling-period $\tau_{2}=m \tau_{1}$ is adopted as the integral-time step length of other controller's DLESO, similarly the new DLESO will be obtained as

$$
\left\{\begin{array}{l}
\eta_{1}(k+1)=\eta_{1}(k)+\eta_{2}(k) \tau_{2}+\beta_{1}^{\prime} e \tau_{2} \\
\vdots \\
\eta_{n}(k+1)=\eta_{n}(k)+\eta_{n+1}(k) \tau_{2}+\beta_{n}^{\prime} e \tau_{2}+b u^{\prime} \tau_{2} \\
\eta_{n+1}(k+1)=\eta_{n+1}(k)+\beta_{n+1}^{\prime} e \tau_{2}
\end{array}\right.
$$

Where $\left[\eta_{1} \eta_{2} \cdots \eta_{n}\right]^{T}$ and $\eta_{n+1}$ are the new estimates of the state variables vector $\left[x_{1} x_{2} \cdots x_{n}\right]^{T}$ and the extended state variable of same plant system (1), respectively.

Having properly selected the parameters of $\beta_{i}$ and $\eta_{i}$, i.e. the two state observers work well. And the control law is given by 


$$
u=\frac{u_{0}-z_{n+1}}{b} \text { and } u^{\prime}=\frac{u_{0}^{\prime}-\eta_{n+1}}{b}
$$

It is known that the plant (1) will be reduced, using the firs DLESO (5), approximately to a unit gain cascaded integrator system which is

$$
\left\{\begin{array}{l}
\dot{z}_{i}=z_{i+1} \\
\dot{z}_{n}=u_{0}
\end{array} \quad(i=1,2, \cdots, n-1)\right.
$$

Similarly, by using DLSEO (6), one can get the new approximate unit gain cascaded integrator which is

$$
\left\{\begin{array}{l}
\dot{\eta}_{i}=\eta_{i+1} \\
\dot{\eta}_{n}=u_{0}^{\prime}
\end{array} \quad(i=1,2, \cdots, n-1)\right.
$$

The discrete-time forms of the above two unit gain cascaded integrators (7) and (8) are obtained as

$$
\left\{\begin{array}{l}
z_{1}(k)=z_{1}(k-1)+z_{2}(k-1) \tau_{1} \\
\vdots \\
z_{n-1}(k)=z_{n-1}(k-1)+z_{n}(k-1) \tau_{1} \\
z_{n}(k)=z_{n}(k-1)+\left(z_{n+1}(k-1)+b u\right) \tau_{1}
\end{array}\right.
$$

and

$$
\left\{\begin{array}{l}
\eta_{1}(k)=\eta_{1}(k-1)+\eta_{2}(k-1) \tau_{2} \\
\vdots \\
\eta_{n-1}(k)=\eta_{n-1}(k-1)+\eta_{n}(k-1) \tau_{2} \\
\eta_{n}(k)=\eta_{n}(k-1)+\left(\eta_{n+1}(k-1)+b u^{\prime}\right) \tau_{2}
\end{array}\right.
$$

, respectively. Because $z_{1}$ and $\eta_{1}$ are both the estimate of $y$, the output of the same plant, so

$$
z_{1}(k)=\eta_{1}(k)
$$

should be guaranteed at every time of calculation. Combining (11), the first equation of (9) and (10) with $\tau_{2}=m \tau_{1}$ to yield

$$
z_{2}(k)=m \eta_{2}(k)
$$

Similarly, combining the second equation of (9) and (10) with above expression (12) to yield

$$
z_{3}(k)=m^{2} \eta_{3}(k)
$$

According to the above approach, repeatedly do the recursive loop calculation, at last the following equations are obtained as 


$$
\left\{\begin{array}{l}
z_{i}(j)=m^{i-1} \eta_{i}(j) \\
u=m^{n} u^{\prime}
\end{array} \quad(i=1,2, \cdots, n+1 \quad j=1,2, \cdots, k \quad k \in \infty)\right.
$$

Finally, combine the above expression (14) with the expression (5) to yield

$$
\left\{\begin{array}{l}
\eta_{1}(k+1)=\eta_{1}(k)+\eta_{2}(k) \tau_{2}+\frac{\beta_{1}}{m} e \tau_{2} \\
\vdots \\
\eta_{n}(k+1)=\eta_{n}(k)+\eta_{n+1}(k) \tau_{2}+\frac{\beta_{n}}{m^{n}} e \tau_{2}+b u^{\prime} \tau_{2} \\
\eta_{n+1}(k+1)=\eta_{n+1}(k)+\frac{\beta_{n+1}}{m^{n+1}} e \tau_{2}
\end{array}\right.
$$

Obviously, compare the above expression (15) with (6), the relation expression is got as follow:

$$
\beta_{i}^{\prime}=\frac{\beta_{i}}{m^{i}} \quad(i=1,2, \cdots, n+1)
$$

Above proof is given from discrete-time digital recursive calculation. From another point of view of differential equation, the theorem 2.1 can also be proven as bellow

Proof: It's well known that the differential forms of LESO for the plant (1), with different integral-time step length $\tau_{1}$ and $\tau_{2}$, are

$$
\left\{\begin{array}{l}
\dot{z}_{1}=z_{2}+\beta_{1} e \\
\dot{z}_{2}=z_{3}+\beta_{2} e \\
\vdots \\
\dot{z}_{n}=z_{n+1}+\beta_{n} e+b u \\
\dot{z}_{n+1}=\beta_{n+1} e \\
y=x_{1}
\end{array}\right.
$$

and

$$
\left\{\begin{array}{l}
\dot{\eta}_{1}=\eta_{2}+\beta_{1}^{\prime} e \\
\dot{\eta}_{2}=\eta_{3}+\beta_{2}^{\prime} e \\
\vdots \\
\dot{\eta}_{n}=\eta_{n+1}+\beta_{n}^{\prime} e+b u^{\prime} \\
\dot{\eta}_{n+1}=\beta_{n+1}^{\prime} e \\
y=x_{1}
\end{array}\right.
$$

respectively. Then using the approach of time-scaling, the above two LESO can be scaled by 
$\frac{\tau_{2}}{\tau_{1}}=m$. Due to properly tracking the output of the same plant (1.1), so $z_{1}$ and $\eta_{1}$, the estimation of the plant's output, which must be uniform. So that will be inferred with $z_{1}\left(\tau_{1}\right)=\eta_{1}\left(\tau_{2}\right)$. From above result, one can be obtained

$$
\left\{\begin{array}{l}
\dot{z}_{1}=\frac{d z_{1}}{d \tau_{1}}=\frac{d \eta_{1}}{d \tau_{2}} \frac{d \tau_{2}}{d \tau_{1}}=m \dot{\eta}_{1} \\
\eta_{2}=\dot{\eta}_{1}, \text { and } z_{2}=\dot{z}_{2} \\
\eta_{2}=m \eta_{2}
\end{array}\right.
$$

Similarly, yielding

$$
\left\{\begin{array}{l}
\dot{z}_{2}=\frac{d z_{2}}{d \tau_{1}}=\frac{m d \eta_{2}}{d \tau_{2}} \frac{d \tau_{2}}{d \tau_{1}}=m^{2} \dot{\eta}_{2} \\
\eta_{3}=\dot{\eta}_{2}, \text { and } z_{3}=\dot{z}_{2} \\
z_{3}=m^{2} \eta_{3}
\end{array}\right.
$$

Continue to recursive iterative compute according to above method, at last one can obtain

$$
\left\{\begin{array}{l}
z_{i+1}=m^{i} \eta_{i+1} \\
\dot{z}_{i+1}=m^{i+1} \dot{\eta}_{i+1}
\end{array} \quad i=1,2, \cdots, n\right.
$$

Lastly, put formula (21) into formula (17) to yield

$$
\left\{\begin{array}{l}
\dot{\eta}_{1}=\eta_{2}+\frac{\beta_{1}^{\prime}}{m} e \\
\vdots \\
\dot{\eta}_{n}=\eta_{n+1}+\frac{\beta_{n}^{\prime}}{m^{n}} e+b u^{\prime} \\
\dot{\eta}_{n+1}=\frac{\beta_{n+1}^{\prime}}{m^{n+1}} e \\
y=x_{1}
\end{array}\right.
$$

Compare the above formula (22) with the anterior formula (17), it's explicit that relation expression can be yielded

$$
\beta_{i}^{\prime}=\frac{\beta_{i}}{m^{i}} \quad(i=1,2, \cdots, n+1)
$$

Which is the same result of (16) obtained from discrete-time inference. And it is known that the characteristic polynomial of LESO (17) is 


$$
s^{n+1}+\beta_{1} s^{n}+\beta_{2} s^{n-1}+\cdots+\beta_{n+1}=s^{n+1}+\sum_{i=1}^{n+1} \beta_{i} s^{n+1-i}
$$

For the sake of simplicity, practicality and tuning, it is proposed that the above polynomial is rewritten as [4]

$$
s^{n+1}+\sum_{i=1}^{n+1} \beta_{i} s^{n+1-i}=\left(s+\omega_{o}\right)^{n+1}
$$

Where $\omega_{o}$ is named as the bandwidth of the LESO, which is the only one parameter needed tuning for the LESO. Properly selecting the $\omega_{o}$ will make the above characteristic polynomial (24) enough stable, which is proven by experiment, practice and theory.

Similarly, the characteristic polynomial of LESO (18) is also got as follow

$$
s^{n+1}+\sum_{i=1}^{n+1} \beta_{i}^{\prime} s^{n+1-i}=\left(s+\omega_{o}^{\prime}\right)^{n+1}
$$

Comprehensively consider and compare the expressions of (23), (25) and (26), the following important equation can be obtained

$$
\omega_{o}^{\prime}=\frac{\omega_{o}}{m}
$$

From above expression (27), it is known that the observer bandwidth is inversely proportional to the sampling-period, if the sampling-period is used as the integral-time step length.

So, a more important approach of selecting parameters of LESO, concluded from the above detailed analysis, is that the new observer bandwidth of LESO $\omega_{o}^{\prime}$ can be easily obtained according to the formula (27), if sampling-period is used as the integral-time step length.

\section{Simulation}

Assume there is a plant which is a second-order system and described in state space as follow

$$
\left\{\begin{array}{l}
\dot{x}_{1}=x_{2} \\
\dot{x}_{2}=M \times \operatorname{sign}\left(\sin \left(2 \pi f_{z} t\right)\right)+230 u \\
y=x_{1}
\end{array}\right.
$$

From previous analysis, it is known that its corresponding LESO is 


$$
\left\{\begin{array}{l}
e=y-z_{1} \\
\dot{z}_{1}=z_{2}+3 \omega_{0} e \\
\dot{z}_{2}=z_{3}+3 \omega_{0}^{2} e+230 u \\
\dot{z}_{3}=\beta_{3} \omega_{0}^{3} \\
y=x_{1}
\end{array}\right.
$$

And the state feedback control laws are

$$
\left\{\begin{array}{l}
u_{0}=k_{p}\left(v_{0}-z_{1}\right)-k_{d} z_{2} \\
u=\left(u_{0}-z_{3}\right) / b
\end{array}\right.
$$

Now, some simulation results are discussed, the "time-scaling" of the plant is defined as $\rho=1 / \sqrt{M}$. From the simulation results shown in Figure 1, 2 and Table I without overshoot, it can be seen that the same sampling-period with deferent "time-scaling" of plants, but the deferent control error is obtained. By further analyzing, the smaller the "timescaling" the larger the control absolute error under the same other conditions can be found.

Table II shows that the cosed-loop system is still steady for all the time if selecting the observer bandwidth according (27) in Section II. Moreover, it demonstrates that as the controller's sampling-time increases, the control absolute error becomes large for the same plant. Conversely, deferent requirements of control precision decide deferent sampling-period of controller.
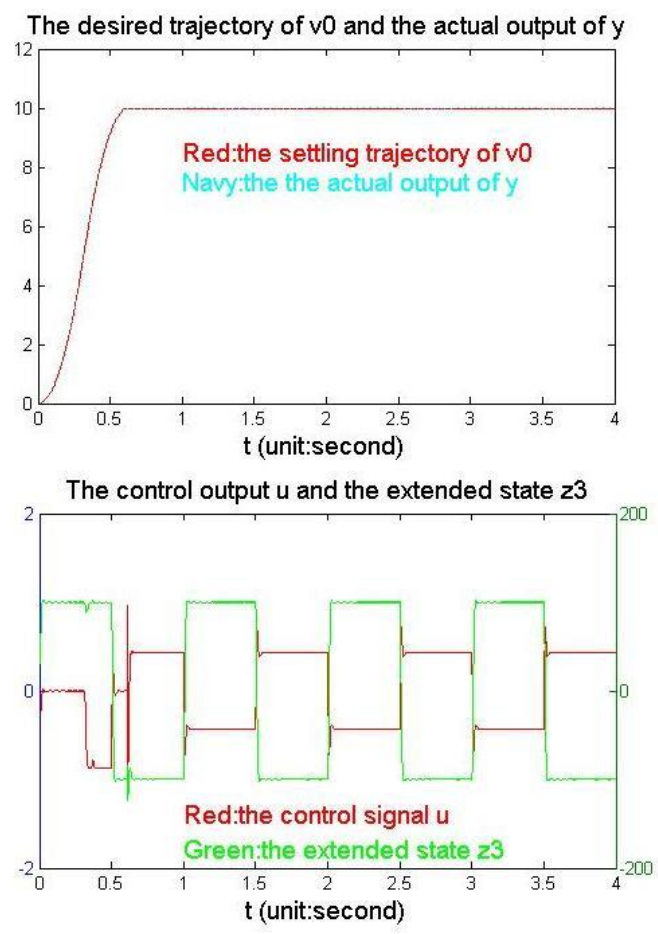

Figure 1. The simulation result with $\mathrm{T}=1 \mathrm{~ms}, \rho=0.1$ 

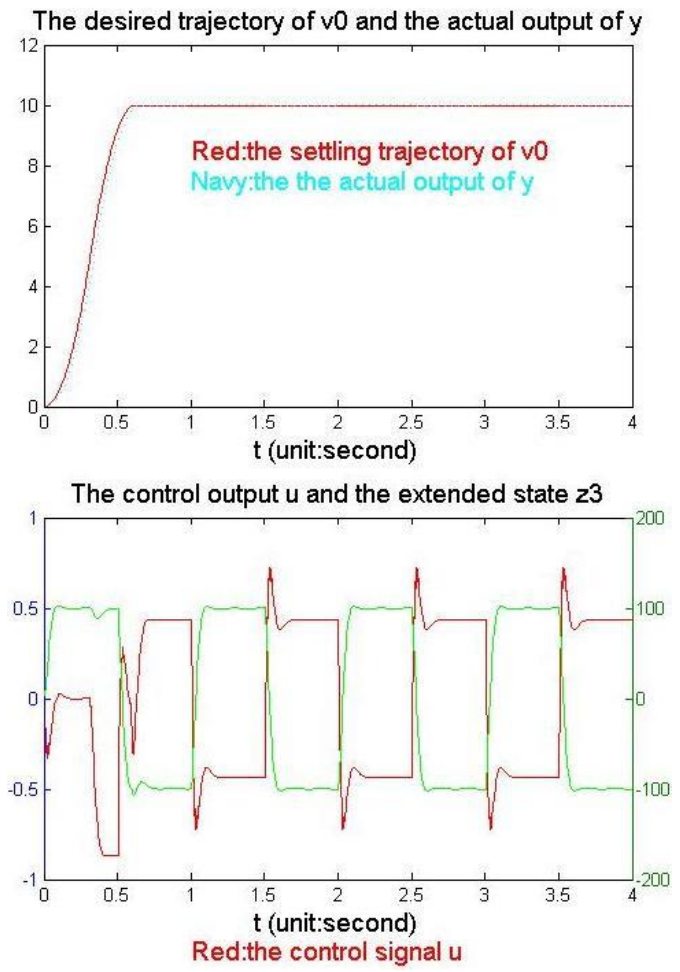

Figure 2. The simulation result with $\mathrm{T}=1 \mathrm{~ms}, \rho=0.2$

Table I. The results of simulation with the same sampling-period of the controller

\begin{tabular}{|c|c|c|c|c|c|}
\hline "time-scaling" & $\begin{array}{c}\text { Sampling-time } \\
(\mathbf{m s})\end{array}$ & $\begin{array}{c}\text { Frequency } \\
\text { of disturbance }\end{array}$ & Set point & $\begin{array}{c}\text { Absolute } \\
\text { error }\end{array}$ & $\begin{array}{c}\text { Settling time } \\
\text { (ms) }\end{array}$ \\
\hline 0.05 & 1.0 & $1-10 \mathrm{~Hz}$ & 10 & 0.0205 & 138 \\
\hline 0.1 & 1.0 & $1-10 \mathrm{~Hz}$ & 10 & 0.0045 & 142 \\
\hline 0.2 & 1.0 & $1-10 \mathrm{~Hz}$ & 10 & 0.0013 & 142 \\
\hline 0.3 & 1.0 & $1-10 \mathrm{~Hz}$ & 10 & 0.00057 & 143 \\
\hline 1.0 & 1.0 & $1-10 \mathrm{~Hz}$ & 10 & 0.000051 & 143 \\
\hline
\end{tabular}

Table II. The results of simulation with the same "time-scaling" of plants

\begin{tabular}{|c|c|c|c|c|c|}
\hline $\begin{array}{c}\text { Observer } \\
\text { bandwidth }\end{array}$ & $\begin{array}{c}\text { Sampling-time } \\
(\mathbf{m s})\end{array}$ & $\begin{array}{c}\text { Frequency } \\
\text { of disturbance }\end{array}$ & Set point & $\begin{array}{c}\text { Absolute } \\
\text { error }\end{array}$ & $\begin{array}{c}\text { settling time } \\
(\mathbf{m s})\end{array}$ \\
\hline 650 & 1.0 & $1-10 \mathrm{~Hz}$ & 10 & 0.0045 & 142 \\
\hline 325 & 2.0 & $1-10 \mathrm{~Hz}$ & 10 & 0.0160 & 145 \\
\hline 163 & 4.0 & $1-10 \mathrm{~Hz}$ & 10 & 0.0820 & 152 \\
\hline 325 & 5.0 & $1-10 \mathrm{~Hz}$ & 10 & 0.1281 & 155 \\
\hline 108 & 6.0 & $1-10 \mathrm{~Hz}$ & 10 & 0.1845 & 156 \\
\hline 93 & 7.0 & $1-10 \mathrm{~Hz}$ & 10 & 0.2511 & 160 \\
\hline 65 & 10.0 & $1-10 \mathrm{~Hz}$ & 10 & 0.5115 & 171 \\
\hline
\end{tabular}




\section{Conclusion}

In this paper, the relation between the observer bandwidth of LESO and the controller's sampling-period has been presented with quantitative description. Properly designing the LADRC based controller according to the above approach detailed described in section II and III, the closed-loop system will always be stable whenever and whatever the sampling-period and the "time-scaling" of the plant system are. And If the sampling-period is used as the integral-time step length, the shorter the sampling-period, the higher the control degree of accuracy is for the same plant. In addition, the simulations above show that the smaller the plant's "time-scaling", the larger the closed-loop control error is for the same samplingperiod, while the closed-loop system is still steady. In other words, in order to improve the control precision, it is proposed that sampling-period is adopted as shorter as possible within limits of software and hardware.

\section{References}

[1] J. Han, "Auto-disturbances-rejection controller and it's application", Control and Decision, vol. 13, no. 1, (1998) (in Chinese), pp. 20-23.

[2] J. Han, "A class of extended state observers for uncertain systems", Control and Decision, vol. 10, no. 1, (1995) (In Chinese), pp. 85-88.

[3] J. Han, "Active Disturbance Rejection Control Technique - the technique for estimating and compensating the uncertainties", Beijing: National Defense Industry Press, (2008) (in Chinese).

[4] Z. Gao, "Scaling and Bandwidth-Parameterization Based Controller Tuning", in Proc. of the 2003 America Control Conference, (2003) June; Denver, CO, USA, pp. 4989-4996.

[5] B. Z. Guo and Z. L. Zhao, "On convergence of tracking differentiator", International Journal of Control, vol. 84, no. 4, (2011) April, pp. 693-701.

[6] J. Q. Han, "From PID to active disturbance rejection control”, IEEE Trans. Ind. Electron, vol. 56, no. 3, (2009) March, pp. 900-906

[7] B. Z. Guo and Z. L. Zhao, "Weak Convergence of Nonlinear High-Gain Tracking Differentiator", IEEE Transactions on Automatic Control, vol. 58, no. 4, (2013) April.

[8] B. Z. Guo and Z. L. Zhao, "On the convergence of an extended state observer for nonlinear systems with uncertainty”, Systems \& Control Letters, vol. 60, (2011), pp. 420-430.

[9] B. Z. Guo and Z. L. Zhao, "On convergence of non-linear extended state observer for multi-input multioutput systems with uncertainty", IET Control Theory and Applications, vol. 6, Issue 15, DOI: 10.1049/ietcta.2012.0123, (2012), pp. 2375-2386.

[10] B. Z. Guo and Z. L. Zhao, "On convergence of the Nonlinear Active Disturbance Rejection Control for MIMO Systems”, SIAM J. CONTROL OPTIM, vol. 51, no. 2, (2013), pp. 1727-1757.

[11] B. Z. Guo and J. J. Liu, "Sliding mode control and active disturbance rejection control to the stabilization of one-dimensional Schrödinger equation subject to boundary control matched disturbance", International Journal of Robust and Nonlinear Control, (2013), DOI: 10.1002/rnc.2977.

[12] B. Z. Guo and F. F. Jin, "Sliding Mode and Active Disturbance Rejection Control to Stabilization of OneDimensional Anti-Stable Wave Equations Subject to Disturbance in Boundary Input", IEEE Transactions on Automatic Control, vol. 58, no. 5, (2013) May.

[13] W. Xue and Y. Huang, "On Frequency-domain Analysis of ADRC for Uncertain System", 2013 American Control Conference, (2013) June 17-19; Washington, DC, USA.

[14] Q. Zheng, L. Q. Gao and Z. Gao, "On Stability Analysis of Active Disturbance Rejection Control for Nonlinear Time-Varying Plants with Unknown Dynamics", Proceedings of the 46th IEEE Conference on Decision and Control, (2007) December 12-14; New Orleans, LA, USA.

[15] G. Tian and Z. Gao, "Frequency Response Analysis of Active Disturbance Rejection Based Control System", 16th IEEE International Conference on Control Applications Part of IEEE Multi-conference on Systems and Control, (2007) October 1-3; Singapore. 


\section{Authors}

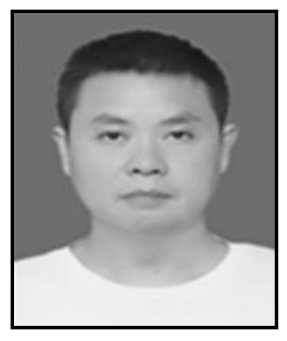

\section{Shangyao Shi}

He received his Master of Engineering in Manufacture of Machinery and Automation (1997) and now is studying at Sichuan University with a major of Measurement and Control Technology and Instrumentation Program for $\mathrm{PhD}$ (2014). Now he is the Chief Engineer in Wuhan HuaGong Laser Engineering corporation, China. His current research interests include Servo Control, Embedded System, Artificial Intelligence and Distributed Control Systems.

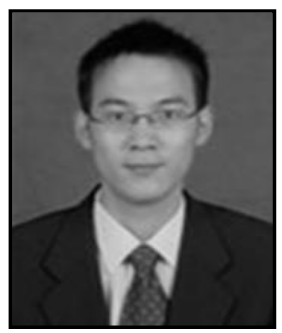

\section{Jun Li}

He received his M.Sc. in Engineering (2008) from southwest University of science and technology. Now he is studying at Sichuan University with a major of Mechatronic Engineering Program for $\mathrm{PhD}$ (2014). His main interesting study on the Electrical discharge machining, Micro Electrical discharge machining and Artificial intelligence used in the mechanical engineering.

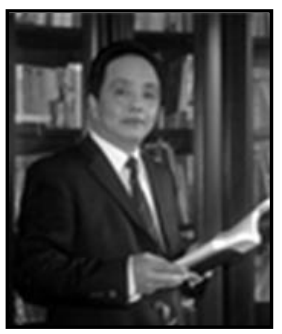

\section{Shiping Zhao}

He received his $\mathrm{PhD}$ in engineering precision instruments and machinery (1991) from Chongqing University. Now he is full professor of manufacturing science and engineering Department, Sichuan University. He makes a great deal research on robot technology and nondestructive test. 
International Journal of Control and Automation Vol.7, No.3 (2014) 